\title{
La gestión de la calidad y su relación con los costos de desechos y desperdicios en las mypes de la confección textil
}

\author{
Elsie Bonilla Pastor \\ Universidad de Lima. Lima, Perú \\ Correo electrónico: ebonilla@correo.ulima.edu.pe
}

Recibido: 29 de octubre de 2014 / Aprobado: 1 de julio de 2015

\begin{abstract}
Resumen: Las micro y pequeñas empresas del Perú, más conocidas como mypes, representan el 99,3\% del total de empresas y su nivel de competitividad es bajo. La inadecuada gestión de la calidad de sus procesos genera desechos y desperdicios que incrementan los costos. La investigación sobre una muestra de 27 mypes de la confección textil concluye que existe una correlación inversa entre la calificación de la gestión de la calidad y los costos por desechos y desperdicios. La investigación se desarrolló durante el periodo 2013-2014.
\end{abstract}

Palabras clave: mypes / gestión de la calidad / desechos y desperdicios / residuos sólidos / costos / textiles

\section{Quality Management and it's Relationship with the Costs of Waste and Scrap in Small Textile Clothing Companies}

AbstRaCT: The MSE's of Peru represent 99,3\% of all enterprises and their competitiveness is low. The inadequate quality management of processes generates waste and scrap which increase costs. The research on a sample of 27 MSE's of textiles concludes that there is a inverse correlation between the rating of the quality management and the cost of waste and scrap. The research was conducted during 2013-2014.

Key words: MSE's / quality management / waste and scrap / solid waste / costs / textiles 


\section{INTRODUCCIÓN}

El rubro textil de la confección es el segundo más importante del sector manufacturero en términos del número de empresas, en el que solo el $0,4 \%$ está compuesto por grandes empresas y el $94,7 \%$ son microempresas. Según la Sunat, se estima que dicho sector está representado por 20755 establecimientos formales, de los cuales el $51 \%$ está ubicado en Lima y Callao, y la PEA relacionada se estima en 139456 habitantes (Ministerio de la Producción, 2011). Un informe presentado el año 2005 por el Instituto Español de Comercio Exterior (ICEX), después de realizar un diagnóstico del rubro textil de la confección, plantea que si bien los tratados de libre comercio firmados con los países del norte representan una oportunidad para las exportaciones textiles, en el Perú existe un sector fragmentado, con presencia de mypes poco competitivas y de frágil condición financiera, lo cual lleva a que las exportaciones se concentren en pocas compañías, generalmente de gran tamaño. La competitividad de aquellas empresas se ve afectada por los niveles de calidad de los productos y servicios, por el precio, así como por la baja inversión tecnológica.

La investigación tuvo como objetivo principal relacionar la gestión de la calidad de las mypes de la confección textil de Lima y Callao con los costos generados por desechos y desperdicios. Quienes conducen las pequeñas y microempresas no son conscientes de la importancia de gestionar adecuadamente la calidad de sus procesos productivos, desde la etapa de diseño hasta que el producto está terminado, perjudicando el cumplimiento de los requisitos de calidad y generando altos niveles de desechos y desperdicios, cuyo valor económico es ignorado; asimismo, la falta de registros y documentación de la gestión impide la búsqueda racional de las causas raíz asociadas y no facilita la mejora continua e innovación de los procesos. En particular, el estudio se halló ante la restricción de tener que cuantificar objetivamente las mermas generadas en las diversas etapas del proceso productivo, en vista de que se carecía de información registrada al respecto.

Deming (1986), el "Padre de la calidad", manifiesta:

el fracaso de la gestión para planificar el futuro y predecir los problemas ha traído como consecuencia un despilfarro de mano de obra, materiales y de tiempo-máquina, todo lo cual incrementa el costo del fabricante y el precio que debe pagar el comprador. (p. XI) 
Asimismo, explica que la causa fundamental de la enfermedad de la industria estadounidense y el consecuente desempleo es el fracaso de la dirección, que no dirige, valga la redundancia, pues quien no vende no puede comprar. La dirección, manifiesta, debe comprender el diseño del producto y del servicio, la adquisición de materiales, los problemas de producción, el control del proceso, y las barreras que privan al trabajador del derecho de estar orgulloso de su trabajo. Se dice que cuando Deming preguntó a 22 operarios de una fábrica ¿por qué sucede que al mejorar la calidad aumenta la productividad?, la respuesta unánime fue... "No hay desperdicios". Al mejorar la calidad se transfieren las horas hombre y las horas máquina malgastadas en la fabricación de producto bueno y a dar un servicio mejor. El resultado es una reacción en cadena: se reducen los costos, se es más competitivo, la gente está más contenta en su labor, hay trabajo y más trabajo. Un esquema que grafica la ruta de lo señalado por Deming se muestra a continuación:

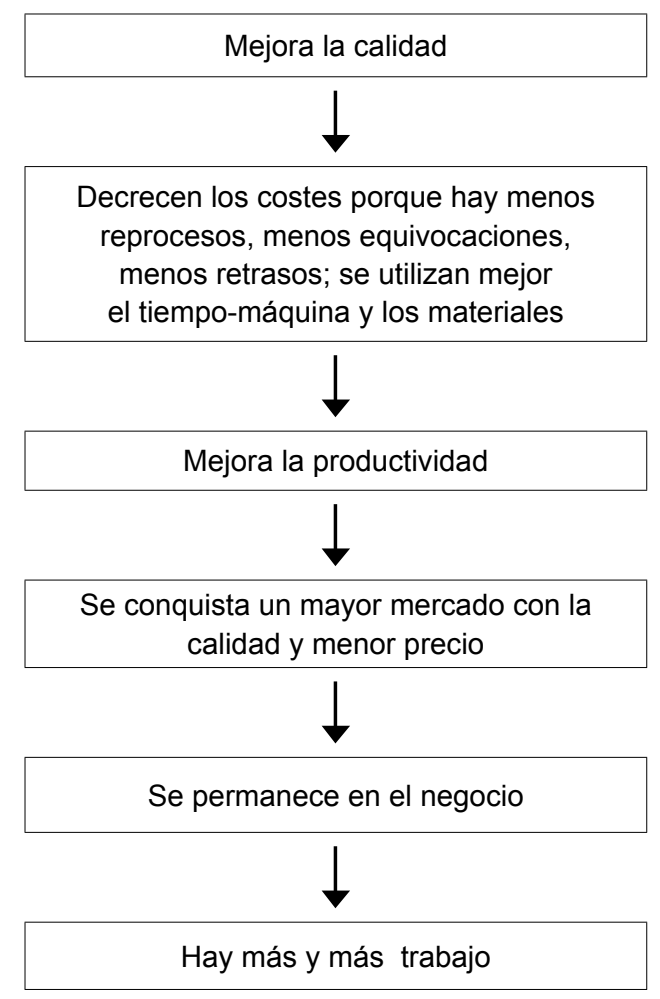

Figura 1. Esquema de la mejora de la calidad Fuente: Deming (1986) 
Tabla 1

Mejoras luego de implementar un sistema de gestión de la calidad

\begin{tabular}{lcc}
\hline Rubro & $\begin{array}{c}\text { Antes de la mejora } \\
\text { (11 \% defectuosos) }\end{array}$ & $\begin{array}{c}\text { Después de la } \\
\text { mejora } \\
\text { (5 \% defectuosos) }\end{array}$ \\
\hline $\begin{array}{l}\text { Coste total } \\
\begin{array}{l}\text { Desembolso por hacer } \\
\text { unidades buenas }\end{array}\end{array}$ & 100 & 100 \\
$\begin{array}{l}\text { Desembolso por hacer } \\
\text { unidades defectuosas }\end{array}$ & 11 & 95 \\
$\begin{array}{l}\text { Número de unidades } \\
\text { defectuosas }\end{array}$ & 11 & 5 \\
\hline
\end{tabular}

Fuente: Deming (1986)

Del análisis de la tabla 1 podemos observar los siguientes beneficios:

- Mejor calidad; el porcentaje de productos defectuosos baja de $11 \%$ a $5 \%$.

- La capacidad de producción se incrementa en un $6 \%$.

- Se reduce el costo unitario de los productos.

- La productividad aumenta en 6,74\%.

La baja calidad significa costes elevados y cuando un gerente comprende la magnitud del problema, es decir pagar dinero por hacer defectos, así como para corregirlos, buscará las maneras de mejorar los procesos y de ayudar a las personas en la línea para que entiendan mejor cómo hacer su trabajo. El coste del reproceso es solo una parte del coste de la mala calidad.

Como lo explica Alberto Alexander (1994), la mala calidad disminuye la productividad a lo largo de toda la línea, y parte del producto defectuoso sale hasta llegar a las manos del cliente. El cliente, disgustado, se lo cuenta a sus amigos. El efecto multiplicador de un cliente disgustado es una de esas cifras desconocidas e incognoscibles, y lo mismo ocurre con el efecto multiplicador de un cliente satisfecho, que atrae más clientes.

A. V. Feigenbaum (citado por Deming, 1986) estimó que entre el $15 \%$ y el $40 \%$ de los costes de fabricación de casi todos los productos estadounidenses que se vendían en su época se debían al desperdicio de esfuerzo humano, de tiempo-máquina y del uso no productivo de las cargas incluidas. 
Mauricio Lefcovich (2004) opina que el sistema Kaizen de mejora continua tiene como uno de sus pilares fundamentales la lucha permanente en la eliminación de desperdicios y despilfarros (mudas, en japonés): una lucha implacable y sin respiro en la necesidad de eliminar los factores generadores de improductividades, altos costos, largos ciclos, costosas y largas esperas, desaprovechamiento de recursos, pérdida de clientes y defectos de calidad; todo lo cual origina la pérdida de participación en el mercado, caída en la rentabilidad y en los niveles de satisfacción de los consumidores. Lefcovich (2004) manifiesta:

una empresa que no controla sus desperdicios, que no tiene noción de ellos, y que por tanto no adopta medidas para prevenirlos o eliminar sus causas gestará productos y servicios de mala calidad, con altos costos y malos servicios, o sea bienes con un bajo valor para los clientes, por lo que ellos no estarán dispuestos a su adquisición o sólo lo harán a un muy bajo precio. (p. 8)

\section{METODOLOGÍA}

El estudio se realizó de manera transversal, descriptiva y correlacional, sobre una muestra representativa de 27 mypes formales de la confección textil de prendas en tejido de punto y tejido plano. La información colectada corresponde al año 2013. El análisis se ha enfocado en la gestión del proceso de producción, conformado por los siguientes subprocesos: corte y habilitado, confección de piezas y armado y acabado.

La metodología utilizada incluyó:

a) Entrevistas con los conductores de las empresas a fin de explicar los objetivos de la investigación y los instrumentos que se van a utilizar para la colecta de información.

b) Aplicación de un primer cuestionario al conductor de la mype o al jefe de producción de la empresa. El instrumento permitió valorizar el nivel de implementación del sistema de gestión de la calidad (SGC) de cada empresa. Los aspectos del SGC por evaluar se han basado en el modelo de gestión ISO 9001:2008 (Bonilla, Díaz, Kleeberg y Noriega, 2010).

c) Aplicación del segundo cuestionario, para cuantificar los desechos y desperdicios generados en cada etapa del proceso productivo y expresar su costo como un porcentaje del costo de producción. La falta 
de registros creó la necesidad de validar las respuestas, con observaciones directas al proceso productivo.

d) Entrevista con expertos (técnica Delphi) para validar algunas conclusiones y brindar mayor soporte al análisis.

El procesamiento de la información y la comprobación de la hipótesis planteada utilizaron los software Minitab y SPSS. También se aplicaron pruebas de normalidad, correlación, regresión y pruebas estadísticas de significancia de Pearson, Spearman, Wilcoxon y la prueba paramétrica t de Student.

\section{RESULTADOS DE LA INVESTIGACIÓN}

A continuación se presentan los principales resultados obtenidos en la investigación.

\subsection{Valoración de la gestión de la calidad de las mypes de la confección textil}

Con relación a la valorización de la gestión de la calidad, el primer cuestionario ponderó el nivel de implementación de los requisitos básicos solicitados por el modelo de gestión ISO 9001:2008; las preguntas se agruparon en los siguientes criterios o factores:

1. Documentación del sistema de gestión de la calidad (X1)

2. Responsabilidad de la dirección (X2)

3. Gestión de los recursos (X3)

4. Gestión de la realización de la prenda textil (X4)

5. Medición, análisis y mejora (X5)

Los resultados se muestran en las figuras 2 y 3 .

Se puede observar que la calificación se halla en el rango de 20 a $75 \%$, y el promedio global es de casi $41 \%$. Se percibió que $35 \%$ de las empresas obtuvieron una valoración por debajo de $30 \%$, otro $35 \%$ estuvo entre 30 y $50 \%$, y el $30 \%$ restante logró una calificación entre 50 y $78 \%$.

Las valoraciones promedio para cada factor se muestran en la figura 3. Se observa que el área con mayor calificación (51,41\%) es el de

1 Organización Internacional de Estandarización (ISO): Norma Internacional ISO 9001: 2008 Sistema de Gestión de la Calidad. 
"Responsabilidad de la dirección". Dicho aspecto califica el compromiso de los conductores de las mypes con el desarrollo e implementación del SGC. El área de menor calificación es el de "Medición, análisis y mejora" (30,96 \%), aspecto que se refiere al esfuerzo de la organización para

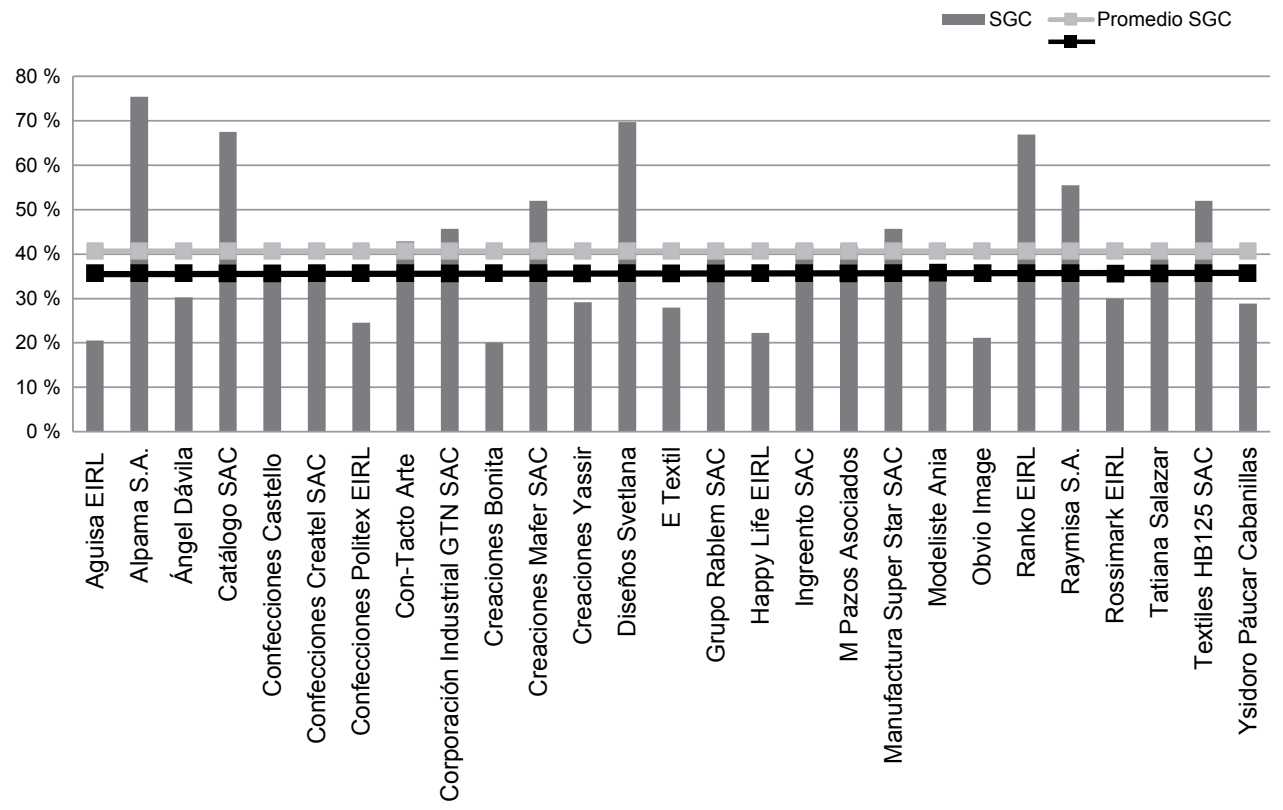

Figura 2. Valoración de la implementación del SGC en cada mype Elaboración propia

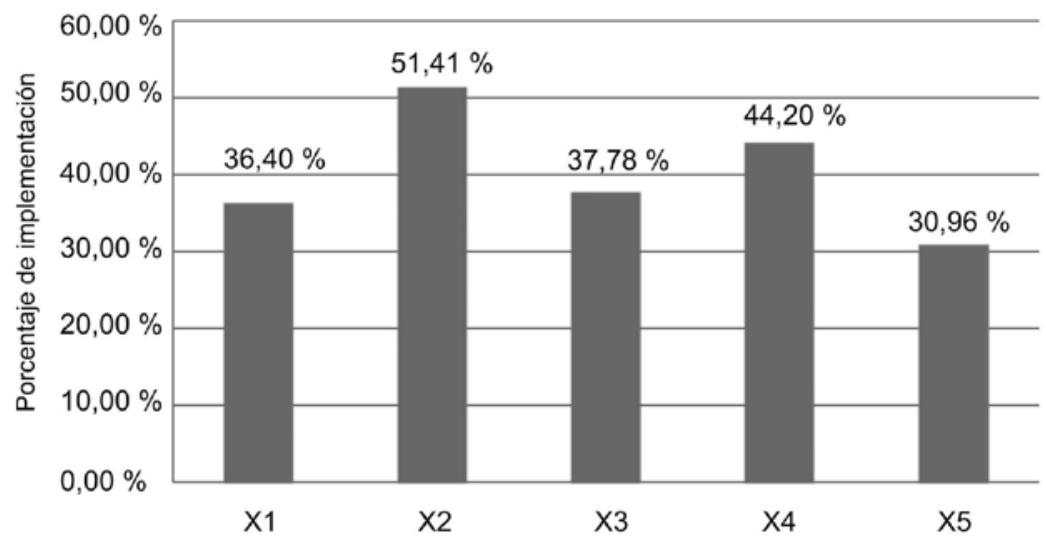

Figura 3. Valoración promedio de los factores del SGC Elaboración propia 
medir, registrar y analizar las incidencias en los procesos productivos que afecten la calidad y el esfuerzo para eliminar las causas raíz y, en consecuencia, promover la mejora continua.

Con relación a la calificación de las preguntas contenidas en cada uno de los cinco aspectos evaluados, estas fueron evaluadas utilizando la escala de Likert, con una puntuación máxima de " 5 ".

Los resultados de la evaluación de las siete preguntas del criterio X1, Documentación del SGC, muestran que el puntaje máximo corresponde a "La existencia de un organigrama y del Manual de funciones y responsabilidades" y el menor puntaje lo alcanza el ítem "La existencia de un Manual de calidad".

El criterio X2, Responsabilidad de la Dirección, se compuso de cinco preguntas. "La dirección facilita la disponibilidad de recursos para el buen desempeño" fue el ítem de mayor calificación, y el de más baja calificación: "La dirección tiene un plan de calidad para lograr los objetivos de calidad".

En cuanto al aspecto Gestión de los recursos (X3), se incluyeron seis preguntas, de las cuales la de más baja calificación fue "La organización elabora e implementa un plan de capacitación para el personal" y la mejor calificada: "La organización determina los edificios, espacios de trabajo, máquinas y equipos necesarios para lograr una adecuada calidad del producto".

Respecto del criterio X4, Gestión de la realización de la prenda textil, conformada por doce preguntas, las de más baja valoración fueron:

- La empresa ha elaborado un plan de control de calidad.

- La empresa mantiene los registros que demuestran la revisión del cumplimiento de los requisitos de calidad en materiales y productos.

- La empresa aplica sistemáticamente encuestas para conocer el nivel de satisfacción de los clientes.

Por otro lado, se pudo observar que entre las preguntas de mayor valoración se encuentran:

- El uso de documentos para la compra de materiales, donde básicamente se describe el producto, la cantidad y el precio.

- El cuidado del proceso de diseño y desarrollo de productos.

- La observancia de las fichas técnicas entregadas por los clientes. 


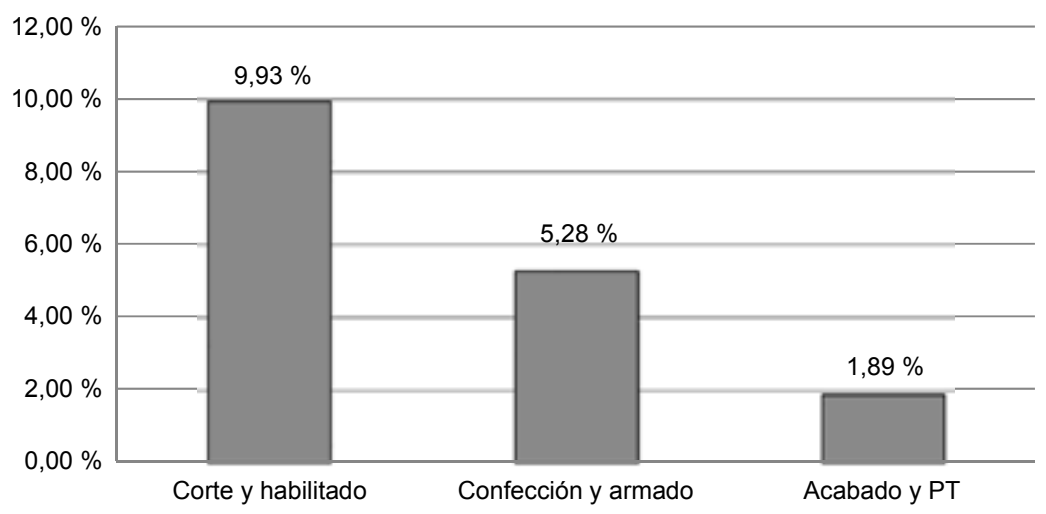

Figura 4. Porcentaje promedio de desperdicios y desechos en las mypes Elaboración propia

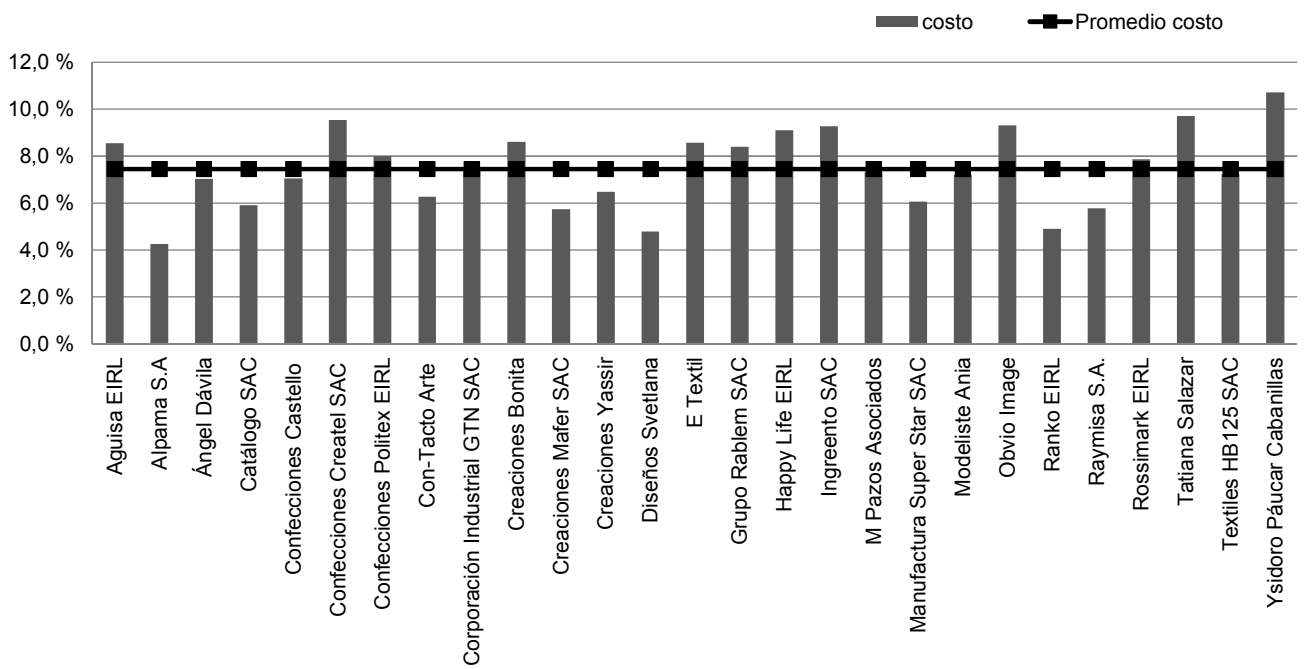

Figura 5. Costos de desperdicios y desechos como porcentaje del costo de producción Elaboración propia

Con relación al factor Medición, análisis y mejora (X5), este fue el aspecto que obtuvo el menor promedio de valoración. Los cinco ítems contenidos en este rubro lograron una calificación menor de 2 puntos, y su promedio general fue de 1.5. Las más bajas calificaciones las obtuvieron las preguntas relacionadas con el seguimiento y la medición de los procesos y la medición de la satisfacción del cliente. 


\subsection{Resultados de la aplicación del segundo cuestionario: contabilización de desechos y desperdicios}

La medición de los porcentajes promedio de desechos y desperdicios en cada etapa del proceso se muestra en la figura 4. Se aprecia que la tasa más alta la tiene el proceso de corte y habilitado $(9,93 \%)$, y la menor es la generada en la etapa de acabado y planchado $(1,89 \%)$.

Posteriormente se calcula el costo representado por los desechos y desperdicios y se le relaciona con el costo de producción correspondiente. Los resultados se grafican en la figura 5 .

Se puede observar que el indicador costo de desechos y desperdicios / costo de producción está comprendido entre 4,3\% y 10,7 \%; el promedio fue de $7,4 \%$.

\subsection{Análisis de la correlación entre la calificación del SGC y los costos de desechos y desperdicios}

La investigación evaluó la normalidad de las variables "Valoración del SGC de las mypes de la confección textil" (X), y la variable dependiente "Costo de desecho y desperdicios, como porcentaje del costo de producción" (Y), para luego establecer la relación entre ambas. Utilizando la prueba de Pearson, y con un alto nivel de significancia, se determina que la relación inversa queda expresada con la función lineal $\mathrm{Y}=10.7-0.0803 \mathrm{X}$.

El coeficiente de determinación $\left(\mathrm{r}^{2}\right)$ es de 0.588 y coeficiente de correlación (r) de 0.7668 .

Se determinó que por cada $1 \%$ de calificación adicional a la gestión de calidad de la empresa, se puede reducir en $0,08 \%$ el costo de producción. En la figura 6 se muestra dicha relación en forma gráfica.

\subsection{Resultados de la reunión con los expertos del sector (técnica Delphi)}

Con la finalidad de validar los resultados obtenidos en la aplicación de los cuestionarios 1 y 2 aplicados a las mypes, se decide aplicar la técnica Delphi; la reunión convocó a 18 expertos provenientes del Ministerio de la Producción, la Cámara de Comercio de Lima, la Corporación Financiera de Desarrollo (Cofide), del Servicio Nacional de Adiertamiento en Trabajo Industrial (Senati), la Sociedad Nacional de Industrias (SNI), la Universidad Nacional de Ingeniería (UNI), de Industrias Textiles 


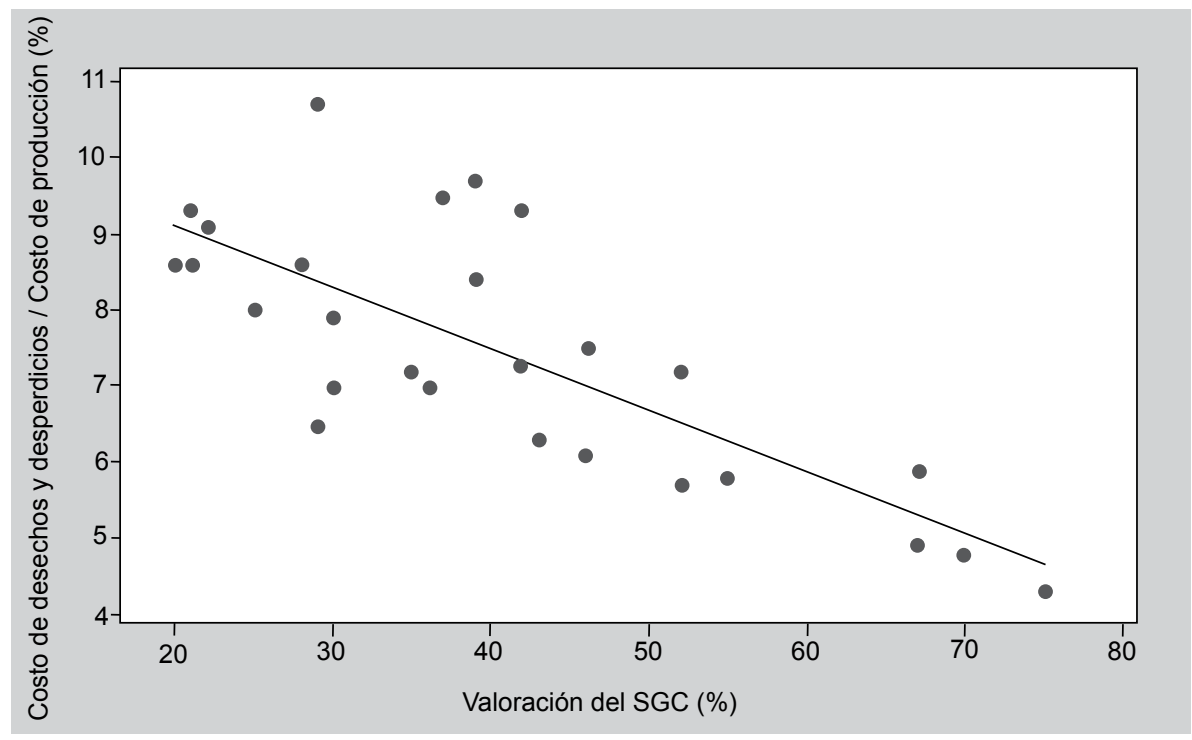

Figura 6. Función de regresión entre la valoración del SGC y costos de desechos y desperdicios

Elaboración propia

de Sudamérica S. A. C. (Itessa), QualityLab, La Molina Calidad Total, entre otros; quienes calificaron la importancia de 11 aspectos vinculados a la gestión de las mypes de la confección textil.

La mayor calificación fue de 4.6 en la escala de Likert y fue asignada a "La eficacia de la gestión de la calidad influye directamente en los niveles de desechos y desperdicios generados en la cadena productiva de las pymes de la confección textil”; asimismo, más del 50 \% opinó que los principales factores de la gestión que determinan la productividad y calidad en las mypes de la confección textil son el compromiso y liderazgo de la dirección, y la competencia y experiencia del personal dedicado a la producción.

\section{CONCLUSIONES}

- Existe una correlación inversa y fuerte entre la valoración (calificación) de la gestión de la calidad y los niveles de costos por desechos y desperdicios generados en las diferentes etapas del proceso productivo de las mypes de la confección textil de Lima y Callao. 
- Los cinco factores del Sistema de Gestión de la Calidad (SGC) fueron encontrados como predictores estadísticamente significativos $(\mathrm{p}<0.05)$ del indicador "costo de desecho y desperdicio/costo de producción". Asimismo, se ha determinado que los factores que muestran un coeficiente de correlación "fuerte" son:

- Documentación del SGC.

- Gestión de la realización de la prenda textil.

- El nivel de implementación de un SGC basado en ISO 9001-2008 en las mypes de la confección textil de Lima y Callao alcanza una calificación promedio de casi $41 \%$, estando el rango de calificación entre $20 \%$ y $75 \%$.

- El factor "Medición, análisis y mejora continua" muestra la más baja calificación (30 \%) con relación a los otros factores del SGC, debido principalmente al perfil profesional de los conductores de las mypes, que no promueven una gestión formal, documentada y cuyas decisiones no se basan en resultados.

- Existe una correlación directa entre el número de trabajadores y la valoración lograda por el SGC, lo cual podría justificarse por una mayor disponibilidad de recursos para capacitación, mejor nivel profesional de los conductores y mayor compromiso con el mercado. En un $40 \%$ de las veces, la calificación del SGC queda explicada por la administración de la capacitación y de las competencias; un empleado capacitado con el objetivo de reforzar sus habilidades, tendrá una mayor cantidad de recursos técnicos y emocionales que le permitirán planificar, ejecutar, controlar y mejorar su trabajo.

\section{RECOMENDACIONES}

- Utilizar la ecuación de regresión entre la valoración del SGC y el indicador de costos de desechos y desperdicios para estimar los costos por desechos y desperdicios en el sector de la confección textil de prendas en las mypes peruanas, y como recurso de futuras investigaciones relacionadas.

- Fortalecer los aspectos de gestión "Documentación del SGC" y "Gestión de la realización de la prenda textil" para reducir, en forma significativa, los desperdicios en el proceso productivo. Un instrumento estratégico de refuerzo sería el uso de indicadores y la herramienta del Balanced Scorecard (Cuadro de Mando Integral), a fin de eva- 
luar sistemáticamente los resultados de desempeño; su diseño puede ser parte de una investigación futura.

- Que el Consejo Nacional para el Desarrollo de la Micro y Pequeña Empresa (Codemype) promueva la implementación de un modelo de gestión de la calidad, como el propuesto por la presente investigación, con el fin de contribuir a la eficacia de la gestión y con ello a la mejora de su competitividad.

- Las universidades, institutos técnicos como el Senati y diferentes gremios empresariales deben ofrecer cursos y talleres que permitan la capacitación de los conductores y empleados de las mypes de la confección textil en relación con la administración de la mejora continua de sus procesos y productos.

- Se sugiere sensibilizar a las mypes de la confección textil sobre las ventajas de la implementación temprana del modelo de gestión, como el que se propone, para evitar que el negocio sea abortado por el mercado en su etapa inicial.

- Las universidades y organizaciones que promueven el desarrollo y competitividad de las mypes en el país deben diseñar un modelo de gestión del talento humano, el cual además de desarrollar las competencias técnicas permite elevar el nivel de inteligencia emocional de los conductores y trabajadores, contribuyendo a crear un mejor clima laboral y calidad de vida en la sociedad, así como a elevar la eficacia y rentabilidad de dichas empresas.

\section{REFERENCIAS}

Alexander, A. (1994). La mala calidad y su costo. Buenos Aires: Addison Wesley Iberoamericana.

Alexander, A. (1995). Aplicación de ISO 9000 y cómo implementarlo. Buenos Aires: Addison Wesley Iberoamericana.

Bonilla, E., Díaz, B., Kleeberg, F., y Noriega, M. T. (2010). Mejora continua de los procesos: Herramientas y técnicas. Lima: Universidad de Lima, Fondo Editorial.

Deming, W. E. (1986). Out of the crisis: quality, productivity and competitive position. Cambridge, MA: Cambridge University Press.

ICEX - Instituto Español de Comercio Exterior. (2005). Informes sectoriales. El sector textil y la confección en el Perú. Recuperado de www.icex.es/icex/es 
Imilce, M. (s. f.). MYPE competitiva, una MYPE con calidad. Guía para entender y evaluar un sistema de gestión de la calidad. Lima: Proyecto USAID/Perú.

ISO - Organización Internacional de Estandarización. (2008). Norma Internacional ISO 9001:2008. Sistemas de Gestión de la Calidad.

Lefcovich, M. (2004). Kaizen - Detección, prevención y eliminación de desperdicios, una estrategia para la reducción de costos. Santa Fe, Argentina: El Cid.

Ministerio de la Producción. (2011). Codemype. Estadísticas. Lima: Produce. Recuperado de www.produce.gob.pe/remype/data/ mype2011.pdf 